\title{
AS POSSIBILIDADES DE USO DA INTERNET NA PESQUISA EM ENFERMAGEM*
}

\author{
THE POSSIBILITIES OF THE INTERNET USE IN NURSING RESEARCH
}

LA POSIBILIDAD DE USO DE LA INTERNET EN LA INVESTIGACIÓN DE ENFERMERÍA

\section{Yolanda Dora Martinez Évora ${ }^{1}$}

RESUMO: O alto nível de conectividade da Internet apresenta oportunidades incomparáveis para o acesso e o compartilhamento da informação. Toda a essência da Internet se resume na transmissão de informações na forma eletrônica com velocidade e confiabilidade. O objetivo deste estudo é apresentar as possibilidades de uso da Internet como ferramenta para o desenvolvimento de pesquisa em Enfermagem e para a divulgação do conhecimento. Trata-se de uma pesquisa de atualização onde são apresentados alguns recursos disponíveis na World Wide Web (www) como em especial os sites de busca, correio eletrônico, acesso à base de dados, fóruns e listas de discussão, transferência de arquivos (FTP), homepages e videoconferência que se tornam indispensáveis e fundamentais quando se pensa em pesquisa. Conclui-se que a Internet é um recurso valioso para quem não tem as informações que precisa ao encontrar um problema na prática. O computador permite às pessoas acharem todo tipo de informação facilmente. Quando a informação está on-line, uma busca adequada pode recuperar a informação que se necessita de forma muito mais rápida do que quando realizada manualmente. $O$ estudo demonstra que é possível o acesso a bases de dados nacionais e internacionais, com busca on-line em tempo real. Além disso, a Internet possibilita o intercâmbio entre pesquisadores de diferentes regiões geográficas em um curto espaço de tempo, por meio da utilização dos recursos disponíveis descritos e muito utilizados para o desenvolvimento da pesquisa em enfermagem.

PALAVRAS-CHAVES: Internet; Enfermagem; Pesquisa em enfermagem.

ABSTRACT: The high level of internet connectivity shows incomparable opportunities for the access and sharing of the information. All the essence of the Internet is summarized in the information and electronic transmission form with speed and reliability. The purpose of this study is to present the possibilities of the Internet use as a tool for the research development in Nursing and for the knowledge transmission. It is an updating research where some World Wide Web (www) available resources are presented. Special attention is given for the search sites, electronic mail, database access, forums and discussion lists, transfer of files (FTP), homepages and videoconferencing. It is concluded that the Internet is a valuable resource for who doesn't have the information that needs when finding a problem in the nursing practice. The computer allows the person to find every type of information easily. When the information is on-line, an appropriate search can recover the information that is needed much faster than when accomplished manually. This study demonstrates that with this tool it is possible the access to national and international data bases, with on-line search, in real time. Besides, the Internet makes possible the exchange among researchers of different geographical areas in a short space of time through the use of the described available resources and very used for the development of the nursing research.

KEYWORDS; Internet; Nursing; Nursing research; Computer.

RESUMEN: El alto nivel de conexión de la Internet muestra las oportunidades incomparables para el acceso y compartiendo de la información. Toda la esencia de la Internet se resume en la información y transmisión electrónica con velocidad y confiabilidad. El propósito de este estudio es presentar las posibilidades de uso la Internet como una herramienta para el desarrollo de la investigación de enfermería y para la transmisión del conocimiento. Es una investigación de actualización dónde se presentan algunos recursos disponibles en el Web (www) como atención especial por los sites de dirección, correo electrónico, el acceso a la base de datos, los foros y la lista de discusión, transferencia de archivos (FTP), homepages y videoconferencia. Se concluye que la Internet es un valioso recurso para quién no tiene la información que necesita al encontrar un problema en la práctica. El computador permite a la persona encontrar cada tipo de información fácilmente. Cuando la información es on-line, una búsqueda apropiada puede recuperar la información que se necesita muy más rápido que cuando logró por mano. Este estudio demostró eso con esta herramienta que es posible el acceso a las bases de datos nacional y las internacionales, con la búsqueda on-line, en el tiempo real. Además, con la Internet es posible el intercambio entre investigadores de áreas geográficas diferentes en un espacio corto de tiempo, a través del uso de los recursos disponibles descritos y muy usados para el desarrollo de la investigación de enfermería.

TERMINOS CLAVES: Internet; Enfermería; Investigación de enfermería; Computador.

\footnotetext{
*Trabalho apresentado no Simpósio Internacional Inovação e Difusão do Conhecimento em Enfermagem, Ribeirão Preto, SP, 2003.

1 Enfermeira. Professora Doutora e Livre-Docente da Escola de Enfermagem de Ribeirão Preto da Universidade de São Paulo. Av. Bandeirantes 3900, Campus Universitário, 14040-902, Ribeirão Preto, SP. e-mail: yolanda @eerp.usp.br
} 


\section{INTRODUÇÃO}

Nos últimos anos tem sido grande o avanço tecnológico nas áreas de computação e comunicação diretamente ligados aos microcomputadores e a rede Internet. Esta permitiu que todos os computadores a ela conectados se interligassem para troca de dados e informação, sem qualquer limitação de tempo e espaço. O alto nível de conectividade da Internet apresenta oportunidades incomparáveis para o acesso e o compartilhamento da informação uma vez que a essência da Internet se resume na transmissão de informações na forma eletrônica com velocidade e confiabilidade.

A Internet - rede mundial de computadores nasceu em 1969 tendo sido idealizada pela Agência de Pesquisas Avançadas do Departamento de Defesa dos Estados Unidos da América (DARPA) e se chamava ARPAnet. Originou-se como um sistema de apoio a uma eventual reação militar em caso de ataque soviético ao território americano (JAMIL \& NEVES, 2000; CALIRI, 1997). Nesta época, a rede era utilizada apenas pelos militares para interligar computadores espalhados pelo país, para o tráfego de dados.

O termo Internet com o significado de interligação de redes de computadores surgiu em 1982, quando a tecnologia ARPAnet deixou de ter uso exclusivo dos militares e passou a ser também utilizada por algumas universidades americanas, sobretudo para o desenvolvimento de pesquisas, inicialmente nos EUA e depois em outros países. No Brasil, a Internet chegou em 1988, entretanto, a primeira ligação à Internet usando o conjunto padrão de protocolos TCP/IP (Transmission Control Protocol/ Internet Protocol) foi realizada pela FAPESP (Fundação de Amparo à Pesquisa do Estado de São Paulo) em fevereiro de 1991 (MANDEL et al., 1997).

Durante cerca de duas décadas a Internet ficou restrita ao ambiente militar, acadêmico e científico. Nos EUA a liberação para a utilização comercial deuse em 1987, entretanto, a sua ampla utilização ocorreu em meados de 1992. No Brasil, os direitos para exploração comercial desta rede foram concedidos apenas em 1994 (ROSA, 1998; CHAGAS, 2003). Desde sua criação, a Internet está, progressivamente, presente no cotidiano da vida das pessoas.

O objetivo deste estudo é apresentar as possibilidades de uso da Internet como ferramenta para o desenvolvimento de pesquisa em Enfermagem e para a divulgação do conhecimento.

\section{A INTERNET E A WORLD WIDE WEB}

A Internet pode ser vista como um "agrupamento de inovações tecnológicas dinâmicas, que pode ser compatível com um contexto de ensino e pesquisa onde se tenha acesso a computadores em rede" (CALIRI, 2002, p.98). Esta rede é formada por milhões de computadores conectados entre si ao redor do mundo, com informações disponíveis 24 horas por dia. Este alto nível de conectividade apresenta oportunidades incomparáveis para promover a intercomunicação e a cooperação em escala global, assim como o acesso e o compartilhamento da informação (MALET, 1997). Toda a essência da Internet, portanto, se resume na transmissão de informações na forma eletrônica com velocidade e confiabilidade.

De acordo com JAMIL \& NEVES (2000), a Internet passou a chamar a atenção do mercado comercial e de negócios principalmente por meio da teia mundial, conhecida como World Wide Web (www) que, por ser considerada fácil de usar, acabou por se constituir hoje em peça importante de contato diversificado entre pessoas e organizações de naturezas variadas.

É comum a pessoa usar os termos Internet e World Wide Web (www) como sinônimos, apesar de não o ser. O www é um componente da Internet que apresenta informação na interface gráfica e permite o acesso a dados diversos na forma de textos, sons, imagens e outros. Trata-se da versão ilustrada da Internet, popularizada a partir de 1994. A teia (www) é, provavelmente, o primeiro passo efetivo na consolidação de uma rede de informações multimídia baseada em hipertextos integrando todo o planeta. Seja qual for a necessidade, é grande a chance de se encontrar a informação ou conteúdo em um servidor de rede (TACHINARDI, 1994), uma vez que os textos e imagens são interligados por meio de palavraschave, tornando a navegação simples e agradável.

Para se localizar um documento ou uma informação na Web usa-se o URL (Universal Resource Location) que identifica o tipo de servidor a ser acessado, o endereço do equipamento onde a informação reside e a sua localização neste equipamento (CHAGAS, 2003). Os endereços de Web sempre se iniciam com http:/l ( Hipertext Transfer Protocol ou protocolo de transferência de hipertexto) e para acessá-los requer um serviço on-line de comunicação comercial. A maioria dos provedores de comunicação da Internet disponibiliza programas de navegação (browsers) tornando a Internet atrativa e acessível para um amplo público.

Hoje a www é a forma padrão de acesso à Internet e principal responsável pela sua facilidade de uso e popularização.

\section{POSSIBILIDADES DE USO DA INTERNET NA PESQUISA}

A Internet é um recurso global que compartilha poder, software e informações pelo computador conectado em rede. Fornece ferramentas de busca, uma infra-estrutura de suporte e uma imensa base de dados digital de informação. Para CALIRI (1997) este avanço da comunicação tem muito a oferecer à Enfermagem, pois pode ser útil não só para os enfermeiros e docentes, mas também aos alunos de graduação na busca de conhecimento, troca de informação e aprendizagem.

Alguns recursos disponíveis na www como em especial os sites de busca, correio eletrônico e 
transferência de arquivos (FTP) tornam-se indispensáveis e fundamentais quando se pensa em pesquisa.

\section{SITES DE BUSCA}

A Internet possibilita diversas maneiras de buscar informações on-line em tempo real. Por meio de sites de busca tais como YAHOO (www.yahoo.com.br), ALTA VISTA ( www.altavista.com), GOOGLE (www.google.com) dentre outros é possível pesquisar assuntos específicos de interesse na forma de artigos completos on-line nas diversas áreas de conhecimento sendo um grande recurso para a realização de pesquisas bibliográficas. Cada site tem seu mecanismo de busca próprio, entretanto a utilização de palavras-chave é uma constante.

Para navegar pela www na busca de informação e de conhecimento, é importante entender o significado de hipertexto para um melhor aproveitamento da pesquisa. Hipertexto é basicamente o mesmo que um texto regular - pode ser armazenado, lido, ou editado - com uma importante diferença: o hipertexto contém ligações (links) dentro do texto para outros documentos. Estes novos documentos, por sua vez, podem ter links e conexões para outros documentos. Dessa maneira, links hipertexto, chamados de hyperlinks, podem criar uma complexa "teia" (web) virtual de conexões e podem referenciar não somente outros textos e mídias, mas também a outros serviços de rede. A linguagem dos documentos publicados na Web é HTML (Hyper Text Markup Language), baseada em diretivas em formato ascii, que permitem definir o formato do documento e as ligações com outros documentos ( hyperlinks).

\section{ACESSO A BASE DE DADOS}

O uso da Internet como recurso para realizar a revisão de literatura vem possibilitar o acesso a diversas Bases de Dados tanto nacionais como internacionais disponíveis hoje em dia, algumas com acesso livre e outras necessitando de assinatura para acesso remoto. Dentre as principais com acesso público pode-se citar:

O Sistema Integrado de Bibliotecas da Universidade de São Paulo (SIBI-USP), considerado a maior rede de bibliotecas universitárias da América Latina. Por meio da Biblioteca Virtual/Internet oferece recursos para a consulta online e links para algumas bases de dados de acesso público por meio do URL: http:// www.usp.br/sibi.

As bases de dados MEDLINE (Medical Literature Analysis and Retrieval System online), mantida pela Biblioteca Nacional de Medicina dos EUA (NLM), engloba informações dos três maiores índices de saúde: Index Medicus (base de dados de publicações médicas); International Nursing Index-INI (Base de dados de publicações em Enfermagem) e o Index to Dental Literature (Base de dados de publicações em Odontologia). Coloca a disposição mais de 4000 periódicos da área biomédica, cobrindo mais de 9 milhões de registros de todo o mundo desde 1966, com predominância da língua inglesa ( ALLEN, 1999). Pode ser consultada no URL: www.nlm.nih.gov

O LILACS (Literatura latino-americana e do Caribe em Ciências da Saúde) é uma base de dados cooperativa do Sistema BIREME que está disponível ao usuário na Biblioteca Virtual em Saúde (BVS) e permite 0 acesso público por meio do URL: http://www.bireme.br.

O ERIC (Educational Resources and Information Center) é a maior fonte de informação existente na área de educação em geral, incluindo educação em enfermagem. Esta base de dados está disponível online desde 1989 e o usuário pode acessá-la, via Internet, no URL: http://eric.syr.edu ou por meio do SIBINet/Biblioteca Virtual.

O SCIELO (Scientific Eletronic Library On-line) é uma biblioteca virtual que disponibiliza textos completos de artigos científicos abrangendo todas as áreas de conhecimento, além de possibilitar o acesso a indicadores de uso e de impacto da literatura nacional. Esta base de dados está disponível na Internet no URL: http://www.scielo.br .

Dentre as bases de dados com acesso regulamentado por assinaturas do serviço on-line pode-se citar dentre outras:

CINAHL (Cumulative Index to Nursing \& Allied Health) é recomendada como busca de primeira linha. Contém as informações de enfermagem mais abrangentes, comparadas com quaisquer outros bancos de dados on-line para fins de uma revisão de literatura para a pesquisa ou para os consumidores de pesquisa. Só estão disponíveis na versão on-line as informações a partir de 1982 encontradas no URL http://www.cinahl.com

CURRENT CONTENTS CONNECT é uma base de dados produzida pelo ISI (Institute for Scientific Information) e a consulta on-line permite visualizar a produção editorial referente à pesquisa acadêmica internacional, por meio de artigos, editoriais, resumos de trabalhos de eventos, livros, resenhas e outros. Este site pode ser consultado por meio do URL: http://www.isinet.com.

\section{CORREIO ELETRÔNICO}

O correio eletrônico (e-mail) enviado e recebido no computador é o recurso mais antigo e mais utilizado da Internet e muito útil no desenvolvimento de pesquisa. Além da vantagem de alcançar o destinatário em qualquer lugar em que estiver para a troca de informações, por meio da utilização do recurso de transferência de arquivos (FTP - File Transfer Protocol) disponíveis automaticamente na www é possível o envio e o recebimento de programas, arquivos e imagens entre os pesquisadores.

Os e-mails podem ser criados em provedores institucionais ou em provedores de serviços comerciais, estes, gratuitos ou não.

Ainda, por meio de e-mail é possível realizar pesquisas do tipo survey utilizando-se de formulário ou 
questionário eletrônico de pesquisa acompanhado do Termo de Consentimento Livre e Esclarecido para a coleta de dados. Este é anexado à mensagem enviada ao sujeito convidado a participar da pesquisa juntamente com as orientações de procedimentos quanto ao preenchimento e retorno das informações solicitadas. Esta forma de coleta de dados é prática, ágil, abrangente e de pouco custo, entretanto não garante o compromisso do sujeito no retorno das informações, uma vez que envolve a transmissão eletrônica de dados e a disponibilidade de acesso fácil à Internet.

A grande vantagem em usar a Internet para a coleta de dados de pesquisa é que estes podem estar estruturados em formato adequado para a pronta análise. Quando se tratar de pesquisa qualitativa, as informações coletadas não precisarão ser transcritas.

\section{FÓRUNS E LISTAS DE DISCUSSÃO EM ENFERMAGEM}

Os fóruns por correio eletrônico e as listas de discussão podem ser usados na Internet como ferramentas para muitas finalidades, dentre elas para a discussão de determinados tópicos ou assuntos de interesse. Essas listas nada mais são do que conjuntos de endereços de e-mail que se formam na Internet quando seus participantes compartilham interesses comuns e usam o correio eletrônico para se comunicar, de qualquer parte do mundo. É uma forma de compartilhar idéias e buscar experiências já vivenciadas. Para se construir uma lista de discussão usa-se um programa especial chamado de processador de lista (listproc) ou servidor de lista (listserv). Os interessados se inscrevem nas listas de interesse e recebem todas as mensagens enviadas pelos membros das listas (CALIRI, 1997). Sendo a Internet uma grande comunidade virtual, ela oferece dezenas de milhares de fóruns e listas de discussão sobre qualquer assunto imaginável, inclusive na área da saúde ou de enfermagem.

\section{TRANSFERÊNCIA DE ARQUIVOS (FTP)}

O FTP (File Transfer Protocol) é um recurso oferecido pela Internet onde é possível a transferência de arquivos entre um computador local e um servidor de FTP remoto, ou seja, pode-se fazer download (copiar) de programas gratuitos de servidores de rede de universidades, de provedores de Internet dentre outros. Os servidores FTP ligados à Internet geralmente permitem sessões anônimas e os arquivos que podem ser copiados estão em área ou diretório público.

\section{VÍDEO CONFERÊNCIA}

Vídeo conferência é uma exposição oral de um determinado tema entre participantes situados em diferentes localizações (cidade, estado, país) durante o qual é possível a comunicação ao vivo transmitindo sons, imagens e dados em tempo real. Pode envolver apenas duas pessoas (ponto a ponto) ou mais (multiponto). A qualidade de transmissão da imagem e som depende da conexão realizada via Internet e dos equipamentos utilizados.

\section{Homepages}

As homepages científicas são páginas da Internet usadas prioritariamente para a divulgação do conhecimento. Normalmente referem-se a Grupos de pesquisas que utilizam deste recurso para disponibilizar os resultados de pesquisas realizadas e para divulgar os novos projetos em desenvolvimento.

\section{REVISTAS ELETRÔNICAS}

Cada vez mais é possível acessar os números on-line de revistas científicas tanto nacionais como internacionais. Por meio do ProBE (Programa de Biblioteca Eletrônica- http://www.probe.br), que reúne em consórcio a FAPESP, USP, UNESP, UNICAMP, UFSCAR, UNIFESP e a BIREME, estão disponíveis 644 títulos de revistas científicas internacionais da área de Ciências da Saúde dos quais 18 são periódicos específicos de enfermagem. A Biblioteca Virtual SCIELO (Scientific Eletronic Library Onlinehttp://www.scielo.br ) abrange uma coleção selecionada de periódicos científicos brasileiros. Conta atualmente com 40 revistas dentre elas está a Revista Latino Americana de Enfermagem que também possui a versão impressa. Como revistas essencialmente eletrônicas brasileiras pode-se citar a Revista Eletrônica de Enfermagem (http://www.fen.ufg.br) e a On line Brazilian Journal of Nursing (http://www.uff.br/nepae/objnursing.htm ).

\section{CONCLUSÕES}

Procurou-se mostrar neste estudo a importância do uso da Internet como ferramenta auxiliar no desenvolvimento de pesquisa e para a divulgação de conhecimento.

De acordo com ARNDT (1992) "para ter conhecimento de toda publicação de real importância para a medicina, seria necessário ler 6.000 artigos por dia". Com a Internet tornou-se possível a aproximação e recuperação de grande quantia de informação publicada nos últimos tempos.

Trata-se de um recurso valioso para quem não tem as informações que precisa ao encontrar um problema na prática. De acordo com CALIRI (2002) a vantagem relativa do uso da Internet para o desenvolvimento de pesquisa é estar associada à obtenção de conhecimento para a prática de enfermagem.

O computador permite as pessoas acharem todo tipo de informação facilmente. Quando a informação está on-line, uma busca adequada pode recuperar a informação que se necessita de forma muito mais rápida do que quando realizada manualmente.

Diante do exposto, observa-se que estando conectado à Internet é possível o acesso a bases de dados nacionais e internacionais, com busca on-line, em tempo real. Além disso, a Internet possibilita o 
intercâmbio entre pesquisadores de diferentes regiões geográficas em um curto espaço de tempo, por meio da utilização de recursos disponíveis como as listas de discussão e o correio eletrônico, estes, muito utilizados para o desenvolvimento da pesquisa em enfermagem.

\section{REFERÊNCIAS BIBLIOGRÁFICAS}

ARNDT, K. A. Information excess in medicine. Arch Dermatol., v. 128, p.1249-56, 1992.

ALLEN, M. Nursing knowledge: access via bibliographic databases. In: THEDE, L. Computers in Nursing: bridge to the future. Philadelphia:Lippincott, 1999. Cap.10, p. 149-170.

CALIRI, M. H. L. Usando os recursos da Internet na Enfermagem. Rev. Latino-Americana de Enfermagem, Ribeirão Preto, v.5, n.1, p.97-102, 1997.

A utilização da pesquisa na prática clínica de enfermagem. Limites e possibilidades. Ribeirão Preto, 2002. 143 p. Tese (Livre-docência). Escola de Enfermagem de Ribeirão Preto da Universidade de São Paulo.

CHAGAS, E. M. P. F. Os novos rumos das aulas tradicionais após o advento da Internet: apresentando algumas discussões. Tecnologia Educacional. Ano 30/31, n. 159/160, p.165-183.

JAMIL, G. L. C.; NEVES,J. T. R. A era da informação: considerações sobre o desenvolvimento das tecnologias da informação. Perspect. Cienc.inf., Belo Horizonte, v.5, n.1, p.41-53, 2000.

MALET, G. Aplicações clínicas da Internet na Medicina. Intermedic. 1997.

MANDEL, A.; SIMON, I.; DELYRA, J. L. Informação: computação e comunicação. Rev.USP,São Paulo, n.35, p. 11-45, 1997.

ROSA, C.A.S. Internet: história, conceitos e serviços. 1. ed. São Paulo (SP): Editora Érica; 1998.

TACHINARDI, U. WWW - A teia de informações. Rev. Bras. Inf. em Saúde, n.6, p. 10-13, 1994.

Texto original recebido em 21/10/04

Publicação aprovada em: 10/12/2004 